\title{
Investigation of Toroidal Acceleration and Potential Acceleration Forces in EAST and J-TEXT Plasmas*)
}

\author{
Fudi WANG ${ }^{1)}$, Bo LYU ${ }^{1)}$, Xiayun PAN ${ }^{1,2)}$, Zhifeng CHENG $^{3)}$, Jun CHEN ${ }^{1,2)}$, Guangming CAO ${ }^{1)}$, \\ Yuming $\mathrm{WANG}^{1)}$, Xiang $\mathrm{HAN}^{1)}$, Hao $\mathrm{LI}^{1)}$, Bin $\mathrm{WU}^{1)}$, Zhongyong $\mathrm{CHEN}^{3)}$, Manfred BITTER ${ }^{4)}$, \\ Kenneth HILL ${ }^{4)}$, John RICE ${ }^{5)}$, Shigeru MORITA ${ }^{6)}$, Yadong LI $^{1)}$, Ge ZHUANG $^{3)}$, Minyou YE ${ }^{2)}$, \\ Baonian WAN ${ }^{1)}$, Yuejiang $\mathrm{SHI}^{7,2)}$ and EAST Team \\ ${ }^{1)}$ Institute of Plasma Physics, Chinese Academy of Sciences, Hefei 230031, China \\ ${ }^{2}$ School of Nuclear Science and Technology, University of Science and Technology of China, Hefei 230026, China \\ ${ }^{3)}$ College of Electrical and Electronic Engineering, Huazhong University of Science and Technology, Wuhan, Hubei \\ 430074, China \\ 4) Princeton Plasma Physics Laboratory, MS37-B332, Princeton, NJ 08543-0451, USA \\ 5) Plasma Science and Fusion Center, Massachusetts Institute of Technology, Cambridge, MA 02139, USA \\ ${ }^{6}$ National Institute for Fusion Science, Toki, Gifu 509-5292, Japan \\ ${ }^{7)}$ Department of Nuclear Engineering, Seoul National University, Seoul 151-742, Korea
}

(Received 25 November 2014 / Accepted 4 May 2015)

\begin{abstract}
Experiments were performed on EAST and J-TEXT for ohmic plasmas with net counter- and co-current toroidal acceleration generated by density ramping-up and ramping-down. Core toroidal rotation is increasing with $T_{\mathrm{e}} / T_{\mathrm{i}}$ increasing in observed region $(r / a \leq 0.3)$. There may be certain correlation between rotation variation and temporal change in density fluctuation intensity. Meanwhile, it is shown that core counter-current toroidal acceleration is gradually increased with the increase of ramp rate in electron density. Direction reversal from co- to counter-current of edge $\mathrm{C}^{4+}$ toroidal rotation is observed by ramp up in electron density. Additionally on EAST, net co-current toroidal acceleration was also formed by LHCD or ICRF. For the current experimental results, toroidal acceleration was between $-50 \mathrm{~km} / \mathrm{s}^{2}$ in counter-current direction and $70 \mathrm{~km} / \mathrm{s}^{2}$ in co-current direction. $E_{\phi}$ may be one of the co-current toroidal forces, which may generate co-current toroidal acceleration, and acts on the plasma as a whole. On the other hand, electron-ion toroidal friction may be one of the countercurrent toroidal forces, which may produce counter-current toroidal acceleration, because electrons move in counter-current direction in order to produce a toroidal plasma current.
\end{abstract}

(c) 2015 The Japan Society of Plasma Science and Nuclear Fusion Research

Keywords: intrinsic rotation, acceleration and toroidal acceleration, density fluctuation, driving and damping forces, toroidal electric field, electron-ion toroidal friction

DOI: $10.1585 /$ pfr.10.3402069

\section{Introduction}

Rotation and velocity shear play important roles in L-H transition [1-5], formation of internal transport barriers (ITBs) [4, 6, 7], suppression of resistive wall modes (RWMs) [8-12], and reducing turbulent losses of heat and particles transport $[4,13-16]$. For ITER and future reactors, NBI may lose the capability of providing long time and strong rotation due to the large machine sizes, higher density and the limitations of beam current. Other methods, which could be used to generate strong plasma rotation and shear in those future devices, are therefore under consideration. This includes taking advantage of the intrinsic rotation, which arises in the absence of external momentum input.

In experimental aspect, intrinsic rotation has been ob-

author's e-mail: fdwang@ipp.ac.cn

*) This article is based on the presentation at the 24th International Toki Conference (ITC24). served in ohmic, LHCD, ICRF, ECRH plasmas [5, 17-24]. But, according to currently experimental results, it is difficult to summarize a simple experimental rule for describing characteristics of intrinsic rotation; meanwhile, the mechanisms for formation of intrinsic rotation are not well understood. Commonly, the possible mechanisms contain driving terms, comprising LHCD, ICRF and ECRH etc., and damping terms including parallel viscosity due to magnetic field ripple, NTV $[25,26]$ and NPV etc. In theoretical aspect, the momentum flux may be considered to be key physics quantity required to confront the mechanisms of generation of intrinsic rotation. Commonly, radial flux of momentum may contain three parts: diffusive, pinch and residual stress term $[27,28]$. The paper simply summarizes the characteristics of toroidal acceleration and toroidal rotation induced by electron density, LHCD and ICRF in EAST and J-TEXT plasmas; meanwhile, the phenomenological understanding on mechanisms of generation of in- 
trinsic rotation is also presented. This paper is organized as follows: section 2 presents experimental results of net toroidal acceleration and rotation on EAST and J-TEXT plasmas. The understanding on mechanisms of generation of net intrinsic toroidal acceleration and rotation is showed in section 3. Finally, results are summarized.

\section{Experimental Results}

The toroidal rotation is measured with X-ray imaging crystal spectrometers (XCS) on EAST and the measured range of toroidal rotation in 2012 and 2014 EAST campaigns is mainly core region of $r / a \leq 0.3$ and $r / a \leq$ 0.4 of plasmas, respectively [29-31]. For lack of an adequate X-ray source for absolute wavelength calibration, the relative change in rotation with respect to that at $9 \mathrm{sec}$ will be discussed. On J-TEXT, the observed region of toroidal and poloidal rotation is commonly edge region (about $0.6<r / a<0.9$ ) in plasmas [32].

Figure 1 shows waveforms of ohmic plasmas in shot No. 42178 on EAST. Plasma current $\left(I_{\mathrm{p}}\right)$ and direction of $I_{\mathrm{p}}$ are $400 \mathrm{kA}$ and in the counter clockwise direction from top view of EAST, respectively. Toroidal magnetic field $\left(B_{\mathrm{T}}\right)$ is $2 \mathrm{~T}$ at $R=1.7 \mathrm{~m}$ and direction of $B_{\mathrm{T}}$ is in the clockwise direction from top view of EAST. Plasma configuration is double null with elongation of about 1.7 during current flattop period. From $3 \mathrm{~s}$ to $5 \mathrm{~s}$, with electron density ramping up, core electron temperature, $T_{\mathrm{e}}(0)$, is gradually decreased from $1.34 \mathrm{keV}$ to $0.81 \mathrm{keV}$; meanwhile, loop voltage, $V_{\text {loop }}$, is gradually increased from $0.63 \mathrm{~V}$ to $1.15 \mathrm{~V}$. From $5 \mathrm{~s}$ to $8 \mathrm{~s}$, with electron density ramping down, $T_{\mathrm{e}}(0)$ is gradually increased from $0.81 \mathrm{keV}$ to $1.21 \mathrm{keV}$; meanwhile, $V_{\text {loop }}$ is decreased from $1.15 \mathrm{~V}$ to $0.70 \mathrm{~V}$.

Shown in Fig. 2 is that core toroidal rotation, in the observed region $(r / a \leq 0.3)$, is gradually decreased in co-current direction following electron density ramping up and following stored energy increasing; meanwhile, core toroidal acceleration $\left(\partial u_{\phi} / \partial t\right)$, in the observed region $(r / a \leq 0.3)$, is about $-10 \mathrm{~km} / \mathrm{s}^{2}$ and in counter-current direction from $3 \mathrm{~s}$ to $5 \mathrm{~s}$. From $5 \mathrm{~s}$ to $8 \mathrm{~s}$, core toroidal rotation, in observed region $(r / a \leq 0.3)$, is increased in co-current direction with electron density ramping down and following stored energy decreasing; meanwhile, core toroidal acceleration $\left(\partial u_{\phi} / \partial t\right)$ is about $5 \mathrm{~km} / \mathrm{s}^{2}$ and in the co-current direction. Based on the rotation velocity and $\mathrm{T}_{e} / \mathrm{T}_{i}$ of $r / a \leq 0.3$ during the period from $3 \mathrm{~s}$ to $8 \mathrm{~s}$, Fig. 3 shows the relationship between toroidal rotatin and $T_{\mathrm{e}} / T_{\mathrm{i}}$, and indicates that the toroidal rotation is increasing with $T_{\mathrm{e}} / T_{\mathrm{i}}$ increasing.

Combining Fig. 2 with Fig. 4 , it is shown that, from $3 \mathrm{~s}$ to $5 \mathrm{~s}$, core toroidal rotation is gradually decreasing in co-current direction following density fluctuation intensity and frequency integrated $S(k)$ increasing; meanwhile, from $5 \mathrm{~s}$ to $8 \mathrm{~s}$, core toroidal rotation is gradually increasing in co-current direction following density fluctuation intensity and frequency integrated $S(k)$ decreasing. But

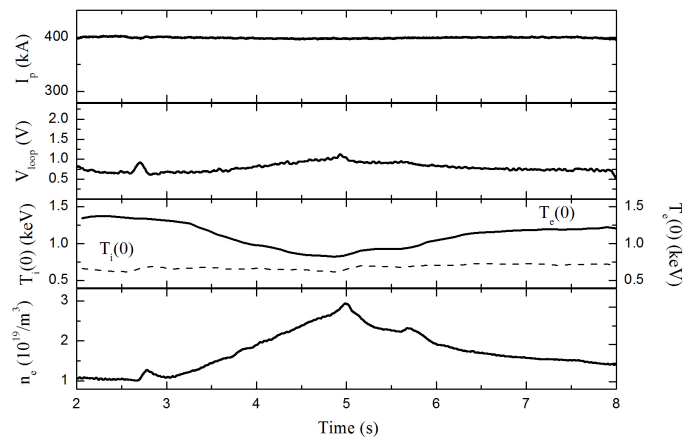

Fig. 1 Time histories of plasma current, loop voltage, core $T_{\mathrm{e}}$, core $T_{\mathrm{i}}$, and line-average electron density in shot No. 42178.

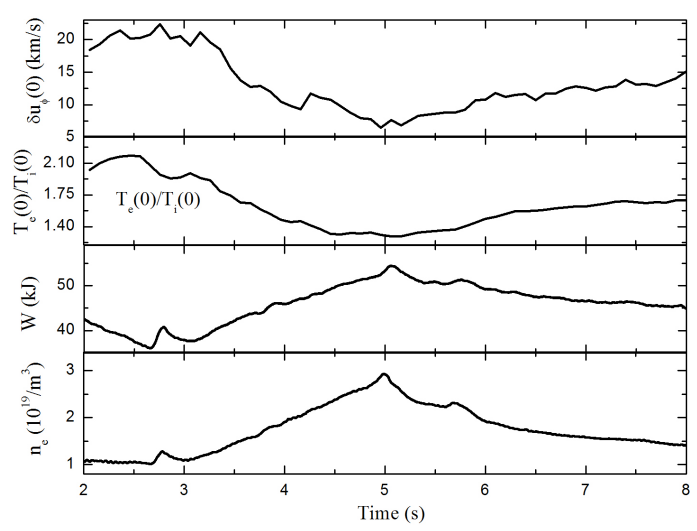

Fig. 2 Time histories of relative toroidal rotation velocity, ratio of electron temperature to ion temperature, plasma stored energy, and line-average electron density in shot No. 42178.

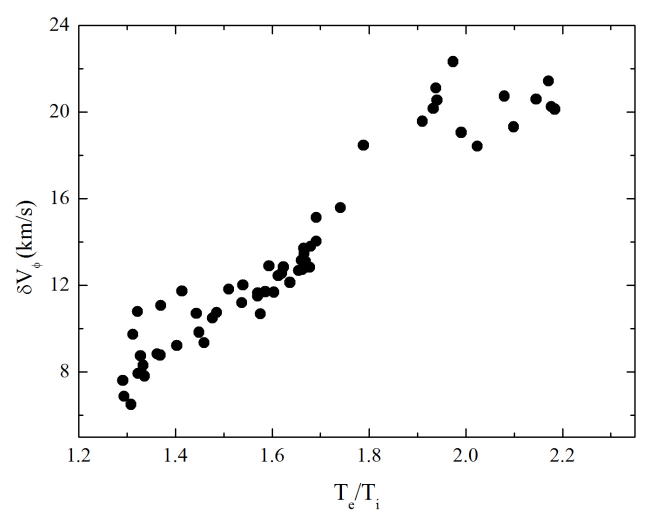

Fig. 3 Relative toroidal rotation versus the ratio of electron temperature to ion temperature in the observed range of $r / a \leq 0.3$.

the $\operatorname{sqrt} S(k) / n_{\mathrm{e}}$ is not obvious changed during the period from $3 \mathrm{~s}$ to $8 \mathrm{~s}$. There may be the certain correlation between core toroidal rotation variation and temporal change in density fluctuation intensity with $k_{r}=10 \mathrm{~cm}^{-1}$.

During electron density of ramping up, relative toroidal rotation $\left(\delta u_{\phi}\right)$ profiles, in observed region $(r / a \leq$ 


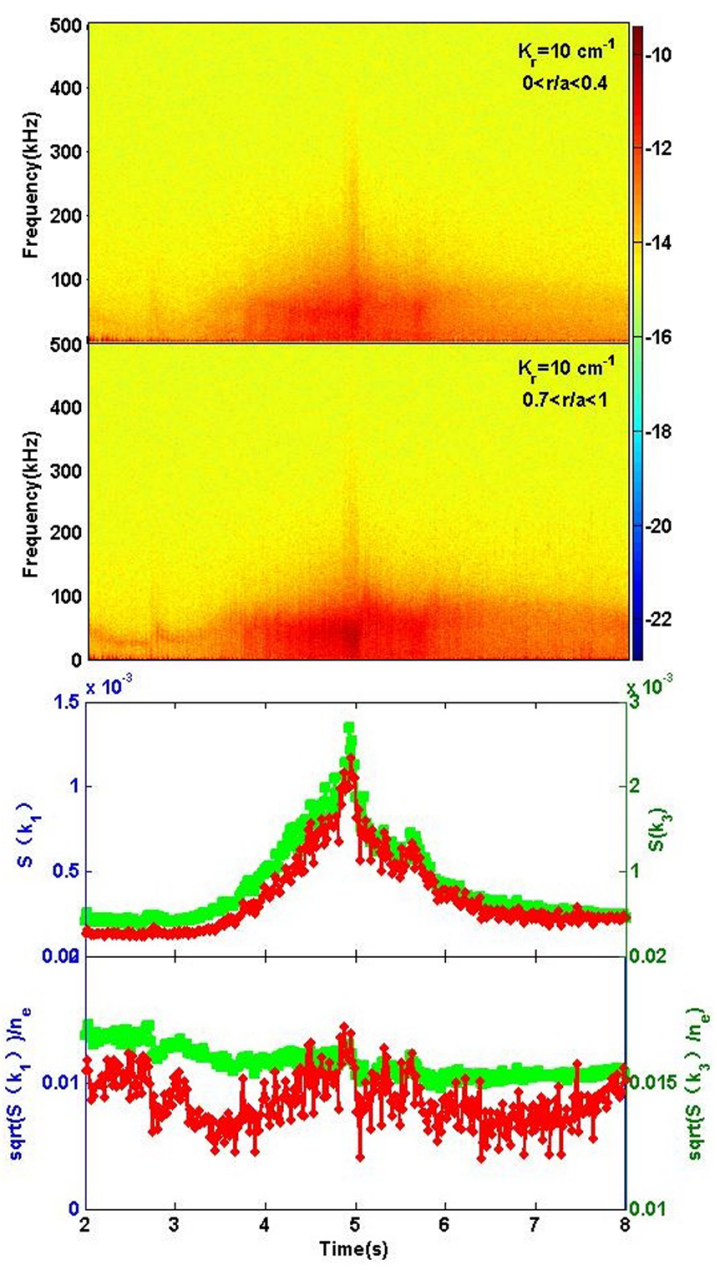

Fig. 4 From top to bottom, figure captions are in turn: time histories of intensity in density fluctuation with $k_{r}=10 \mathrm{~cm}^{-1}$ in $0<r / a<0.4$ and $0.7<r / a<1$ region, respectively; time histories of the frequency integrated $S\left(k_{1}\right)$ (green line) and $S\left(k_{3}\right)$ (red line) in $0<r / a<0.4$ and $0.7<r / a<$ 1 region, respectively; time histories of sqrt $\left(S\left(k_{1}\right)\right) / n_{\mathrm{e}}$ (green line) and sqrt $\left(S\left(k_{3}\right)\right) / n_{\mathrm{e}}$ (red line) in $0<r / a<0.4$ and $0.7<r / a<1$ region, respectively. The spectra power is in units of $\mathrm{dB}$.

0.3 ), are flat and gradually decreased in co-current direction. Ion temperature profiles, in observed region $(r / a \leq$ 0.3 ), are also flat and are not obvious changed. Electron density and temperature profiles are shown in Fig. 5. During electron density of ramping up, electron density profiles and electron density gradient are gradually increasing; On the contrary, electron temperature profiles and electron temperature gradient are gradually decreasing.

Modification of toroidal rotation by ramp rate in electron density $\left(\mathbf{a}_{n_{\mathrm{e}}}=\partial n_{\mathrm{e}} / \partial t\right)$ has been observed over a range of electron densities and plasma currents in ohmic plasmas on EAST. Shown in Fig. 6 is relationship between core toroidal acceleration $\left(\mathbf{a}_{\phi}(0)=\partial u_{\phi}(0) / \partial t\right)$ of observed range $(r / a \leq 0.3)$ and ramp rate of electron density. It is shown that core counter-current toroidal acceleration is gradually increased with the increase of ramp rate in electron density.

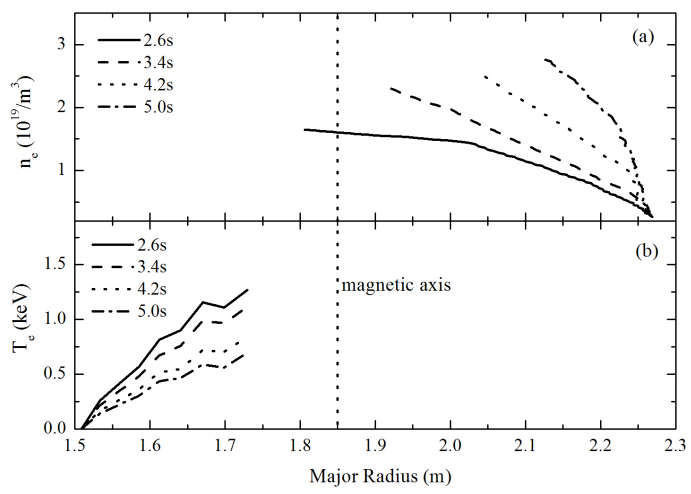

Fig. 5 Profiles of $n_{\mathrm{e}}$ and $T_{\mathrm{e}}$ in shot No. 42178. The magnetic axis is at $R=1.85 \mathrm{~m}$.

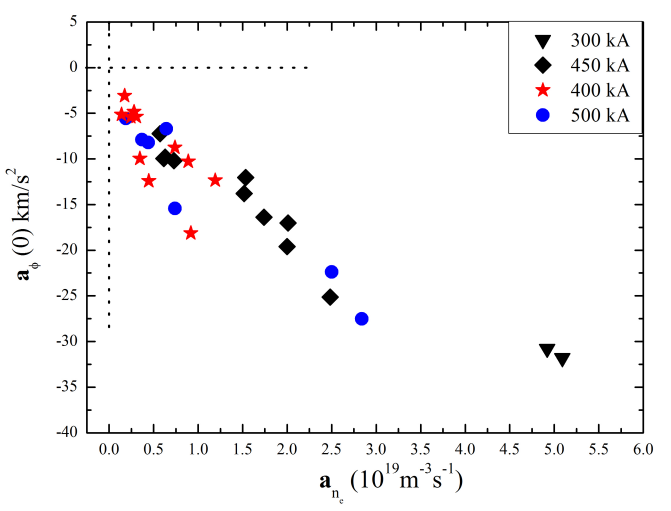

Fig. 6 The toroidal acceleration versus electron density ramp rates. For toroidal acceleration, "+" and "-" represents co- and counter- current direction, respectively. For ramping rate of electron density, "+" and "-" represents ramp up and down, respectively.

In ohmic plasmas on J-TEXT, direction reversal from co- to counter-current of edge $\mathrm{C}^{4+}$ toroidal rotation is observed by ramp up in electron density. The reversal density is commonly $1.6 \times 10^{19} \mathrm{~m}^{-3}$ with $I_{\mathrm{p}}$ of $160 \mathrm{kA}$ and $B_{\mathrm{T}}$ of $1.8 \mathrm{~T}$ at $R=1.05 \mathrm{~m}$. Counter- and co-current toroidal acceleration is generated by electron density ramping-up and ramping-down, respectively. The edge counter-current toroidal acceleration $\left(\mathbf{a}_{\phi}(\right.$ edge $)=\partial u_{\phi}($ edge $\left.) / \partial t\right)$ is increasing with density ramp rate increasing; meanwhile, edge co-current toroidal acceleration is increasing with density decline rate increasing. In the experiments, maximum coand counter-current toroidal acceleration, in the observed region, is $20 \mathrm{~km} / \mathrm{s}^{2}$ and $-50 \mathrm{~km} / \mathrm{s}^{2}$, respectively. Poloidal rotation of $\mathrm{C}^{4+}$ and $\mathrm{C}^{2+}$, in observed range, is not changed during density ramping up and ramping down, so poloidal acceleration is $0 \mathrm{~km} / \mathrm{s}^{2}$.

On EAST, modification of core toroidal acceleration and rotation in $r / a \leq 0.4$ region by $\mathrm{LHCD}$ at $2.45 \mathrm{GHz}$ and $4.6 \mathrm{GHz}$ has been observed over different lower hybrid powers, electron densities, plasma currents and toroidal magnetic fields. Both $2.45 \mathrm{GHz}$ and $4.6 \mathrm{GHz}$ LHCD can induce co-current toroidal acceleration and a co-current 
change of core toroidal rotation of $r / a \leq 0.4$; meanwhile, core toroidal acceleration and rotation is gradually increasing with lower hybrid power increasing. In the experiments, maximum co-current toroidal acceleration and rotation change is $70 \mathrm{~km} / \mathrm{s}^{2}$ and $45 \mathrm{~km} / \mathrm{s}$ at almost no obvious change in $n_{\mathrm{e}}$, respectively. With the injection of LHCD at $2.45 \mathrm{GHz}$ and $4.6 \mathrm{GHz}$, both $\delta u_{\phi}$ and $T_{\mathrm{e}}$, in the observed region of $r / a \leq 0.4$, are increased with keeping their profiles remained; meanwhile, modification of the toroidal rotation develops on a time scale of about $300 \mathrm{~ms}$. Commonly, $T_{\mathrm{i}}$ profile and $T_{\mathrm{i}}$ gradient in observed region is not changed with injection of the LHCD.

Under the injection of ICRF condition on EAST, modification of core toroidal acceleration and rotation in $r / a \leq$ 0.4 region by ICRF at $27 \mathrm{MHz}, 34 \mathrm{MHz}$ and $35 \mathrm{MHz}$ has been observed over different ICRF powers, electron densities, plasma currents and toroidal magnetic fields. All the $27 \mathrm{MHz}, 34 \mathrm{MHz}$ and $35 \mathrm{MHz}$ ICRF can induce cocurrent toroidal acceleration and a co-current change in the core toroidal rotation in $r / a \leq 0.4$ region; meanwhile, core toroidal acceleration and rotation are gradually increasing with increasing ICRF power. In the experiments, maximum co-current toroidal acceleration and rotation change is $40 \mathrm{~km} / \mathrm{s}^{2}$ and $35 \mathrm{~km} / \mathrm{s}$ at almost no obvious change in $n_{\mathrm{e}}$, respectively. With the injection of ICRF, $\delta u_{\phi}, T_{\mathrm{e}}$ and $T_{\mathrm{i}}$, in observed region of $r / a \leq 0.4$, are gradually increasing with keeping their profiles remained; meanwhile, modification of the toroidal rotation develops on a time scale of about $500 \mathrm{~ms}$.

\section{Discussion and Conclusions}

Figure 3 shows the relationship between toroidal rotatin and $T_{\mathrm{e}} / T_{\mathrm{i}}$, and indicates that the toroidal rotation is increasing with $T_{\mathrm{e}} / T_{\mathrm{i}}$ increasing. There may be a certain correlation between origin of toroidal rotation and $T_{\mathrm{e}} / T_{\mathrm{i}}$ (Temperature ratio effects) associated with suppressing the certain turbulent modes (ITG) [33]. Under without large density variation condition, LHCD and ICRF can induce cocurrent toroidal acceleration, it means that, during LHCD and ICRF injection period, net toroidal force is generated. Before discharge in tokamak plasmas, $\mathbf{u}_{\phi}$ is $0 \mathrm{~km} / \mathrm{s}$, but, with plasma current gradually established, $\mathbf{u}_{\phi}$ is gradually formed and commonly is not $0 \mathrm{~km} / \mathrm{s}$. This means that, in toroidal direction, ions are collectively accelerated by net toroidal acceleration which is generated by net toroidal force resulting from both toroidal driving and damping forces. The co-current toroidal driving forces may generate co-current toroidal acceleration and rotation. Toroidal electric field $\left(E_{\phi}\right)$, which can be obtained by $U_{\phi} /(2 \pi R)\left(U_{\phi}\right.$ is loop voltage and measured by voltage loop, $R$ is plasma major radius), is usually in co-current direction and acts on plasma as a whole and persists for the entire discharge period, so $E_{\phi}$ is commonly one of co-current toroidal driving forces. Electron-ion toroidal friction, $\left(R_{\mathrm{ei}}\right)_{\phi}$, may be one of counter-current toroidal forces. Because electrons move in counter-current direction in order to produce a toroidal plasma current, so $\left(R_{\mathrm{ei}}\right)_{\phi}$ imposed on ions is in countercurrent direction. In the future research, the intensity of detailed toroidal driving and damping forces will be calculated according to experimental data of ohmic, LHCD and ICRF plasmas.

In summary, experiments on EAST and J-TEXT were executed for ohmic plasmas with counter- and co-current $\mathbf{a}_{\phi}$ generated by $n_{\mathrm{e}}$ ramping-up and ramping-down within a factor of three density variation. In EAST The toroidal electric field was increased (decreased) during the density ramp-up (down) from $0.05 \mathrm{~V} / \mathrm{m}$ to $0.1 \mathrm{~V} / \mathrm{m}$. Although $T_{\mathrm{i}}$ is remained, $T_{\mathrm{e}} / T_{\mathrm{i}}$ is varied from 2.1 to 1.3 , which evolves in a similar way to that in $\mathbf{a}_{\phi}$. It is found that the toroidal acceleration in the counter-current direction $\left(-2.5 \mathrm{~km} / \mathrm{s}^{2} \sim-32 \mathrm{~km} / \mathrm{s}^{2}\right)$ is roughly proportional to the density ramp-up rate up to $5 \times 10^{19} \mathrm{~m}^{-3} / \mathrm{s}$. On J-TEXT similar toroidal acceleration is also confirmed. Furthermore, direction reversal from co- to counter-current of edge $\mathrm{C}^{4+}$ toroidal rotation is observed by ramp up in electron density. Poloidal rotation of $\mathrm{C}^{4+}$ and $\mathrm{C}^{2+}$, in observed range, is not changed during density ramping up and ramping down. Under a small density variation condition, LHCD or ICRF effects on $\mathbf{a}_{\phi}$ is investigated. On EAST, It is observed that $\mathbf{a}_{\phi}$ in the co-current direction is induced and increased with injection power increasing. $\mathbf{a}_{\phi}$ is $70 \mathrm{~km} / \mathrm{s}^{2}$ for LHCD, and $40 \mathrm{~km} / \mathrm{s}^{2}$ for ICRF. In both devices, the toroidal acceleration was observed between $-50 \mathrm{~km} / \mathrm{s}^{2}$ in counter-current direction and $70 \mathrm{~km} / \mathrm{s}^{2}$ in cocurrent direction by density ramp condition or additional $\mathrm{rf}$ heating in the range of LHW and ICF. No poloidal acceleration was confirmed.

\section{Acknowledgements}

This work was supported by National Magnetic Confinement Fusion Science Program of China (2012GB101001 and 2013GB112004), Natural Science Foundation of China (11305212 and 11175208), JSPSNRF-NSFC A3 Foresight Program in the field of Plasma Physics (11261140328) and Brain Korea 21 Program for Leading Universities \& students (BK21 PLUS).

[1] K.C. Shaing and E.C. Crume, Phys. Rev. Lett. 63, 2369 (1989).

[2] R.J. Groebner, K.H. Burrell and R.P. Seraydarian, Phys. Rev. Lett. 64, 3015 (1990).

[3] K. Ida et al., Phys. Rev. Lett. 65, 1364 (1990).

[4] P.W. Terry, Rev. Mod. Phys. 72, 109 (2000).

[5] I.H. Hutchinson et al., Phys. Rev. Lett. 84, 3330 (2000).

[6] R.C. Wolf, Plasma Phys. Control. Fusion 45, R1 (2003).

[7] J.W. Connor et al., Nucl. Fusion 44, R1 (2004).

[8] A. Bondeson and D.J. Ward, Phys. Rev. Lett. 72, 2709 (1994).

[9] E.J. Strait et al., Phys. Rev. Lett. 74, 2483 (1995).

[10] R. Betti and J.P. Freidberg, Phys. Rev. Lett. 74, 2949 (1995).

[11] A.M. Garofalo et al., Phys. Rev. Lett. 82, 3811 (1999). 
[12] L.J. Zheng, M. Kotschenreuther and M.S. Chu, Phys. Rev. Lett. 95, 255003 (2005).

[13] G.G. Craddock and P.H. Diamond, Phys. Rev. Lett. 67, 1535 (1991).

[14] T.S. Hahm, Phys. Plasmas 1, 2940 (1994).

[15] T.S. Hahm and K.H. Burrell, Phys. Plasmas 2, 1648 (1995).

[16] K.H. Burrell, Phys. Plasmas 4, 1499 (1997).

[17] A. Bortolon et al., Phys. Rev. Lett. 97, 235003 (2006).

[18] J.E. Rice et al., Nucl. Fusion 47, 1618 (2007).

[19] C. Angioni et al., Phys. Rev. Lett. 107, 215003 (2011).

[20] J.E. Rice et al., Nucl. Fusion 51, 083005 (2011).

[21] J.E. Rice et al., Phys. Rev. Lett. 107, 265001 (2011).

[22] A. Ince-Cushman et al., Phys. Rev. Lett. 102, 035002 (2009).
[23] J.E. Rice et al., Nucl. Fusion 49, 025004 (2009).

[24] Y.J. Shi et al., Phys. Rev. Lett. 106, 235001 (2011).

[25] K.C. Shaing, Phys. Plasmas 10, 1443 (2003).

[26] K.C. Shaing, M.S. Chu and S.A. Sabbagh, Plasma Phys. Control. Fusion 51, 075015 (2009).

[27] P.H. Diamond et al., Nucl. Fusion 49, 045002 (2009).

[28] O.D. Gurcan, P.H. Diamond and T.S. Hahm, Phys. Rev. Lett. 100, 135001 (2008).

[29] F.D. Wang et al., J . Korean Phys. Soc. 59, 2734 (2011).

[30] B. Lu et al., Rev. Sci. Instrum. 83, 10E130 (2012).

[31] B. Lyu et al., Rev. Sci. Instrum. 85, $11 \mathrm{E} 406$ (2014).

[32] Z.F. Cheng et al., Rev. Sci. Instrum. 84, 073508 (2013).

[33] E.J. Doyle et al., Nucl. Fusion 47, S18 (2007). 